\title{
Modelación de demanda de transporte urbano en Costa Rica, de Emme a TransCAD
}

\section{Urban transportation demand modelling in Costa Rica, from Emme to TransCAD}

Ing. Leonardo Castro Rodríguez. M.E., M.B.A.

Escuela de Ingeniería Civil, Universidad de Costa

Rica, Costa Rica

leonardo.castro@ucr.ac.cr

\section{Ing. Gabriela Picado Aguilar}

Laboratorio Nacional de Materiales y Modelos

Estructurales, LanammeUCR, Universidad de Costa

Rica, Costa Rica

gabriela.picadoaguilar@ucr.ac.cr

\author{
Ing. Stephan Rodríguez Shum \\ Laboratorio Nacional de Materiales y Modelos \\ Estructurales, LanammeUCR, Universidad de \\ Costa Rica, Costa Rica \\ stephan.rodriguez@ucr.ac.cr
}

Fecha de recepción: 30 de agosto de 2017 / Fecha de aprobación: 13 de noviembre 2017

\section{RESUMEN}

En julio de 2016 la Escuela de Ingeniería Civil de la Universidad de Costa Rica y el Laboratorio Nacional de Materiales y Modelos Estructurales iniciaron el proyecto de investigación Modelo de Demanda de Transporte Urbano de la Gran Área Metropolitana en Plataforma TransCAD (MDTUCR). En este artículo se describe el proceso de migración de las bases de datos y relaciones analíticas, de Emme2 a TransCAD, del modelo de demanda de transporte urbano de la Gran Área Metropolitana (GAM), calibrado en el Plan Regional Urbano de la Gran Área Metropolitana (PRUGAM) en el 2007. La migración de la base de datos contempló tanto la red de carreteras como la red de transporte público, así como la información relacionada con el transporte público y las variables demográficas y socioeconómicas asociadas al modelo. En el caso de la migración de las relaciones analíticas del modelo de demanda de transporte urbano, se describe la migración por etapas. En términos generales, se resume la metodología para exportar la información de Emme2 hacia TransCAD, las consideraciones tomadas para validar el proceso de migración y el modelo integrado de demanda implementado en TransCAD. Como conclusión principal del artículo se resalta que, de forma preliminar, el modelo de demanda de transporte urbano del PRUGAM pudo migrarse de Emme2 a TransCAD mediante diversos ajustes de forma, pero sin cambios relevantes en las relaciones analíticas. Los métodos presentes en TransCAD son similares o presentan mejoras respecto a los utilizados en Emme2 para el PRUGAM, por lo que se logró replicar el modelo de forma satisfactoria.

PALABRAS CLAVES: Demanda, modelación, transporte urbano, Gran Área Metropolitana.

\section{ABSTRACT}

The Modelo de Demanda de Transporte Urbano de la Gran Área Metropolitana en Plataforma TransCAD (MDT-UCR) project began in 2016, conceived as a research project of the University of Costa Rica, based on a partnership between the Civil Engineering School (EIC) and the National Laboratory of Testing Materials and Structural Models (LanammeUCR). The present article describes the database migration of the urban transportation demand model, from Emme2 to TransCAD, generated as a result of the Regional Urban Plan for the Great Metropolitan Area of Costa Rica (PRUGAM). The databases include the roads and transit networks, the transit system's operational characteristics and the socioeconomic variables used for transportation demand modeling. Each step of the demand model migration process is described. This article covers the methodology used for exporting data generated in Emme 2 to TransCAD and the considerations used for validating both the databases' migration process and the results obtained from the migrated urban transportation demand model in TransCAD. The main conclusion drawn from the migration process is that, even though some changes in the structure of the data had to be made, the urban transportation demand model was successfully migrated from Emme 2 to TransCAD, without changes to its contents. The analytical methods included in TransCAD are similar to those used in Emme2, so the results obtained by the model were successfully replicated.

KEYWORDS: Demand, modeling, urban transportation, Great Metropolitan Area. 


\section{INTRODUCCIÓN}

El modelo para el análisis de la demanda de transporte urbano de la GAM de Costa Rica existente en el 2016 fue generado en el Plan Regional Metropolitano de la GAM (PRUGAM) de 2008 y desarrollado con ayuda del software Emme2. Emme2 es un programa de modelación de demanda de transporte que incorpora, de las cuatro etapas convencionalmente utilizadas para analizar demanda (generación, distribución de viajes, distribución modal, asignación de flujos), únicamente la etapa de asignación de flujos. Sin embargo, por medio de la programación en lenguajes macro del propio software, fue posible desarrollar las primeras tres etapas del modelo mediante procesamiento matricial de datos.

Para el año 2017, la versión del software Emme comercializada por INRO corresponde a Emme4, la cual ya trae incorporadas las cuatro etapas del modelo y puede ser utilizada a través de una interfaz gráfica. Sin embargo, en Costa Rica, el modelo en Emme2 nunca fue totalmente actualizado a la nueva versión y las bases de datos no recibieron mantenimiento. Por lo tanto, en julio de 2016 se inició el proyecto de investigación Modelo de Demanda de Transporte Urbano de la Gran Área Metropolitana (GAM) en Plataforma TransCAD (MDT-UCR), como una colaboración entre la Escuela de Ingeniería Civil (EIC) y el Laboratorio Nacional de Materiales y Modelos Estructurales (LanammeUCR) de la Universidad de Costa Rica. El objetivo principal del proyecto consistió en migrar, del programa Emme2 a TransCAD, el modelo de demanda de transporte urbano de la GAM desarrollado en el Plan Regional Urbano de la GAM (PRUGAM), así como su actualización y calibración según las condiciones prevalecientes en 2017.

El presente artículo surge como uno de los resultados del MDTUCR, el cual tiene como fin explicar el proceso de migración de las bases de datos y las relaciones analíticas, las dificultades encontradas y los procesos llevados a cabo para cumplir con el propósito general de contar con un modelo de transporte urbano en plataforma TransCAD.

\section{OBJETIVO}

El objetivo principal del presente artículo consiste en documentar el proceso de migración, de Emme 2 a TransCAD, de las bases de datos y relaciones analíticas necesarias para el adecuado funcionamiento del modelo de demanda de transporte urbano.

\section{MODELO DE DEMANDA DE TRANSPORTE URBANO EN COSTA RICA}

\section{Generalidades}

Desde el 2003 se utilizó el software Emme2 en Costa Rica para modelar la demanda de transporte urbano de la GAM. Los modelos en esta versión del software se generaban a través de códigos en lenguaje de macro, los cuales eran activados por medio de una ventana de comandos. Esto representaba una ventaja porque brindaba gran flexibilidad con respecto a los modelos utilizados para la aplicación de las primeras tres etapas, ya que a través del lenguaje macro se podía programar cualquier tipo de procedimiento matricial requerido para su aplicación. De igual forma, esta facilidad tenía sus desventajas, pues requería de un conocimiento avanzado del lenguaje de programación de los macros utilizado por Emme2.

La base de datos acoplada a Emme2 surgió como resultado del PRUGAM. Esta base de datos fue generada en el año 2007 y su desarrollo incluyó diferentes actividades y componentes: una encuesta de hogares específicamente diseñada para el modelo de demanda, conteos de tránsito y de viajes en transporte público, proyecciones de población y otras variables socioeconómicas.

Para efectos del proyecto MDT-UCR se utiliza el software TransCAD, el cual permite la modelación de demanda de transporte urbano integrada con Sistemas de Información Geográfica (SIG). TransCAD tiene incorporadas las cuatro etapas tradicionales de modelación de demanda de transporte urbano: generación de viajes, distribución de viajes, selección modal y asignación de flujos. Adicionalmente, es capaz de leer diferentes tipos de archivos asociados a SIG.

En términos de modelación, TransCAD cuenta con una interfaz gráfica que permite al usuario acceder a cada una de las etapas del proceso de modelación. Cada etapa tiene ecuaciones típicas asociadas, de manera que el usuario puede incorporar los coeficientes de las ecuaciones correspondientes o introducir los valores mediante una planilla de cálculo. Si no se tienen las ecuaciones calibradas, se pueden elegir, de tablas ya incorporadas al sistema TransCAD, valores típicos según las diferentes características demográficas de la región en estudio. Esto no se recomienda en el caso de la aplicación del modelo en Costa Rica, ya que estos valores están calibrados a partir de casos de estudio en Estados Unidos y no necesariamente coinciden con los patrones de movilidad en Costa Rica.

Para la modelación en el proyecto MDT-UCR se utilizó como punto de partida el esquema de zonas y la base de datos del PRUGAM, la cual incluía la red de carreteras con la respectiva caracterización de las vías a nivel de nodos y enlaces, rutas de transporte público con el trazado de los recorridos e información de los servicios provistos por ruta, así como las ecuaciones e 
información socioeconómica necesarias para la modelación de demanda de transporte urbano.

\section{ASPECTOS TÉCNICOS DE LA MIGRACIÓN DEL MODELO DE DEMANDA DE TRANSPORTE URBANODELAGRANÁREAMETROPOLITANA: DE EMME A TRANSCAD}

\section{Base de Datos en Emme2}

La base de datos de PRUGAM está compuesta por la red de carreteras, la información relacionada a los servicios de transporte público, datos demográficos, datos socioeconómicos y los métodos analíticos calibrados para las cuatro etapas de la modelación. Para la migración del modelo se incluyeron las 424 zonas internas y 14 zonas externas definidas en el Estudio de Oferta y Demanda de Transportes de la GAM incluido en PRUGAM (2007), para las cuales ya se tenían los valores demográficos, socioeconómicos y las variables de modelación asociadas a todos los pares origen-destino.

\section{Red de Carreteras}

La red de carreteras se encontraba caracterizada a través de nodos y enlaces. Los nodos están georreferenciados y los enlaces se codifican a partir de la ubicación de los nodos. Los nodos y enlaces son una representación gráfica de las intersecciones y carreteras, respectivamente, sobre las cuales el modelo asigna los viajes en vehículo privado y transporte público. En los archivos de nodos y enlaces se incluyó información asociada a la longitud, velocidad permitida, capacidad de la vía, cantidad de carriles y sentido del flujo vehicular, información base que caracteriza las condiciones existentes de la red vial de la GAM.

\section{Transporte Público}

En la base de datos se incluyeron 431 rutas de transporte público urbano de la GAM. La codificación de los sistemas de transporte público corresponde al trazado de cada ruta, generado a partir de los nodos de la red de carreteras, lo cual permite visualizar el recorrido de cada una de las rutas del sistema. Además del trazado, se incluyó el nombre y número de la ruta, la velocidad promedio de operación, la capacidad por tipo de vehículo, el tiempo de espera en parada y el período de arribo. Estos datos caracterizan las condiciones existentes de los servicios de transporte público masivo en la GAM, en modalidad autobús y tren.

\section{Variables Socioeconómicas y para Modelación}

Se incluyeron en el modelo aquellas variables socioeconómicas consideradas significativas en la determinación de la demanda de viajes urbanos, tales como el ingreso por hogar, la posesión vehicular, la población por zona, el tamaño del hogar y la proporción de personas por hogar que se dedican a los tres propósitos determinados para el modelo: trabajo, estudio y otros. Con base en estas variables se desarrollaron las ecuaciones de producción de viajes, así como las tasas de atracción para cada una de las zonas del modelo, según cada uno de los propósitos indicados.

Las ecuaciones de selección modal utilizaron como insumo la posesión vehicular, así como otras variables asociadas al costo de uso de los dos modos de transporte considerados (autobús y vehículo privado), tales como el costo y consumo de combustible y las tarifas por servicio de transporte público.

De manera complementaria, se determinaron variables específicas necesarias para la etapa de distribución de viajes. Esta etapa requiere de una matriz de impedancia o de tiempos de viaje y los factores de fricción, parámetros que permiten ajustar el modelo basado en costos asociados a cada par origendestino, y usando como referencia la matriz de viajes observados según una encuesta de hogares. Estas variables se determinaron para cada uno de los tres propósitos de viaje analizados (trabajo, estudio, otros).

\section{Migración de las Bases de Datos a TransCAD}

Red de Carreteras y Transporte Público

Las redes de carreteras y transporte público generadas a partir de PRUGAM, fueron introducidas al programa Emme2 mediante archivos de texto con formato ".in", siguiendo los protocolos establecidos del software para la codificación de estos archivos. TransCAD tiene incorporada una rutina que permite importar y procesar los archivos con extensión “.in”, siempre que estos sigan los protocolos de codificación establecidos en Emme2. Sin embargo, es necesario que tanto los archivos de la red de carreteras, formada por nodos y enlaces, como el de transporte público, compuesto por rutas, se encuentren ubicados en una misma carpeta al momento de la migración. La red de transporte público estaba codificada a partir de los nodos y enlaces de la red de carreteras, por lo que no tenía coordenadas geográficas y no podía ser migrada de forma independiente.

Producto de la migración de los archivos base se generaron cuatro capas, las cuales incluyen los enlaces y nodos de la red de carreteras y las rutas y paradas de transporte, con sus respectivos atributos y características determinados en el PRUGAM. Dentro de la base de datos de PRUGAM, no se definió la ubicación exacta de las paradas de cada ruta de transporte público, por 
lo que en el modelo cualquier nodo dentro del trazado de la ruta es considerado una parada. Para efectos de la modelación macroscópica de la demanda, esto no es considerado una limitación, ya que permite observar los patrones de flujo de viajes en transporte público sin considerar las restricciones de oferta del sistema, es decir, las paradas establecidas.

Se verificó la ubicación de los nodos y enlaces para asegurar que los centroides, nodos y tramos de carretera estuvieran correctamente ubicados y con la escala correspondiente. TransCAD facilitó la verificación de la ubicación de estos elementos, ya que tiene incorporada la opción de cargar mapas tales como OpenStreetMap, Google Maps o Google Satellite en conjunto con las capas de nodos y enlaces. Sin embargo, fue necesario modificar las coordenadas de los nodos del sistema proyectado Lambert Norte al sistema geográfico WGS84, de manera que la red generada calzara correctamente con la visualización de la aplicación OpenStreetsMaps utilizada por TransCAD.

Para validar la migración se verificó la cantidad de enlaces, velocidades, capacidades, cantidades de carriles y sus respectivas direcciones. Sin embargo, los parámetros de cantidad de enlaces y direcciones de flujo presentaron variaciones al ser migradas. La cantidad de enlaces en Emme2 es cercana a 25.000, pero al migrar la red a TransCAD se obtuvieron aproximadamente 13.000 enlaces. Esta condición surgió por los siguientes motivos:

- Emme2 requiere especificar como enlaces independientes cada sección de vía que presente características diferentes por sentido, ya sea cantidad de carriles, capacidad o velocidad.

- Para modelar en Emme2, los consultores decidieron contar cada sentido de un enlace como un enlace distinto, por lo que una vía de dos sentidos en Emme2, equivale a dos enlaces.

- TransCAD unifica automáticamente los enlaces que comparten la misma ubicación, y genera un único enlace bidireccional con la información correspondiente a cada sentido, aún cuando presenten características diferentes por sentido de circulación.

Este proceso automático de unificación generó la necesidad de revisar los valores de velocidad, cantidad de carriles y capacidad por sentido de cada enlace contra el indicador de dirección de TransCAD, de forma que la información del resto de variables fuera congruente con la direccionalidad de los enlaces y no generara errores al modelar.
Para confirmar que la red de transporte público fue migrada de forma correcta, se verificó tanto el código de ruta como los trazados, de forma que fueran congruentes con los recorridos de cada servicio. Además, se revisó que los atributos de las rutas fueran migrados de manera congruente con lo establecido en PRUGAM. En términos de la red de carreteras y de sistemas de transporte público, se comprobó que existe compatibilidad entre los sistemas Emme2 y TransCAD, y la migración fue realizada de manera satisfactoria.

\section{Variables Socioeconómicas y para Modelación}

Cada una de las variables socioeconómicas utilizadas en el PRUGAM se encontraba en un archivo "in" independiente. Sin embargo, se pudieron migrar todos los archivos de forma simultánea al guardarlos dentro de la misma carpeta. Las variables socioeconómicas utilizadas en el modelo de demanda, fueron:

- Población

- Personas por hogar

- Proporción trabajadores por hogar

- Proporción estudiantes por hogar

- Proporción otros por hogar

- Ingreso familiar

- Atracción relativa trabajadores por zona

- Atracción relativa estudiantes por zona

- Atracción relativa otros por zona

- Posesión vehicular por hogar

Estas variables se agruparon en un solo archivo, con forma de cuadro, al ser migradas a TransCAD, ya que la modelación en este software se facilita al referirse a un único archivo que contenga todas las variables.

Otras variables para modelación que se migraron con el mismo procedimiento, fueron:

- Matriz de tiempos de viaje entre pares origen - destino.

- Tabla de factores de fricción por propósito.

- Matriz de relación de costo percibido de viaje por transporte público entre costo percibido de viaje por vehículo privado.

Estas variables fueron almacenadas como matrices o cuadros según corresponde. La posesión vehicular fue utilizada como 
vector en Emme2; sin embargo, en TransCAD se requiere en forma de matriz, por lo que fue convertida a dicho formato manteniendo el valor de posesión vehicular fijo para los orígenes, pues ahí es donde se producen los viajes.

\section{Migración de Relaciones Analíticas del Modelo de Deman- da de Transporte Urbano}

Para migrar el modelo de demanda de transporte urbano de la plataforma Emme2 a TransCAD hubo que recurrir a procedimientos adicionales a la migración directa de los archivos “.in”. Las consideraciones y procesos realizados por etapa, así como la generación del modelo en lenguaje en lote (batch), se detallan a continuación.

\section{Generación de Viajes}

Producto de la incorporación de los archivos “.in”, se tenían las variables necesarias para calcular las producciones de viajes para los tres propósitos de viaje (trabajo, estudio, otros) con base en las ecuaciones del PRUGAM. Sin embargo, las ecuaciones de generación establecidas por defecto en TransCAD consideran únicamente ecuaciones logit binario o de regresión lineal, mientras que las ecuaciones de generación determinadas en PRUGAM no siguen ninguna de estas dos formas, por lo que no podían ser determinadas a través de la interfaz de producciones. Por esta razón, fue necesario realizar el cómputo de producciones mediante la planilla de cálculo que forma parte de TransCAD, cuya interfaz es similar a la de Excel.

En las ecuaciones 1, 2 y 3 se muestra el cálculo de producción de viajes por zona y para cada propósito (trabajo, estudio y otros), respectivamente.

$$
\begin{array}{ll}
P_{t}=\frac{p_{a}}{h} *\left(0,35983 * T_{h}+0,04827 * I\right) & r^{2}=0,54 \\
P_{e}=\frac{p_{a}}{h} *\left(0,16222 * E_{h}+0,02797 * I\right) & r^{2}=0,54 \\
P_{o}=\frac{p_{a}}{h} *(0,01112 * h+0,01622 * I) & r^{2}=0,05
\end{array}
$$

donde:

$P_{t}:$ producción de viajes con propósito trabajo [viajes/hogar en 2 horas pico a.m.]

$P_{e}$ : producción de viajes con propósito estudio [viajes/hogar en 2 horas pico a.m.]

$P_{o}$ : producción de viajes con otros propósitos[viajes/hogar en 2 horas pico a.m.]

$P_{a}$ : población por zona para el año a [personas]

$h$ : tamaño del hogar [personas/hogar]
$T_{h}$ : trabajadores en el hogar [número de trabajadores/hogar]

$E_{h}$ : estudiantes en el hogar [número de estudiantes/hogar]

I: ingreso familiar estandarizado [ingreso familiar/salario mínimo vigente]

Las atracciones relativas de viajes por cada zona se anexaron al cuadro de viajes calculados con base en las ecuaciones de producción, ya que TransCAD requiere que ambas se encuentren en el mismo cuadro con el fin de efectuar el proceso de balanceo. Dicho proceso se realizó manteniendo las producciones fijas, debido a que hay más confiabilidad en la estimación de las producciones que en la estimación de las atracciones. Adicionalmente, las ecuaciones de producción dan como resultado viajes de personas, mientras que las tasas de atracción se consideraron en términos de proporciones, por lo que debían ser estandarizadas en términos de viajes de personas. Como resultado del balanceo se obtienen los viajes atraídos en cada una de las zonas consideradas en el estudio.

\section{Distribución de Viajes}

TransCAD permite replicar el modelo de gravedad generado en el PRUGAM, acotado a producciones. La interfaz del software permite establecer la matriz de tiempos de viajes como el costo generalizado entre los pares origen-destino e introducir los valores de impedancia en formato de cuadro, generados a partir de la función de impedancia por propósito que se ilustra en el gráfico de la Figura 1. Esta figura muestra el comportamiento de las funciones de impedancia por propósito. Dado que para la modelación en Emme2 se contaba con los archivos "in” con los factores de fricción discretos, por propósito y en intervalos de un minuto, esta información pudo ser incorporada directamente al modelo en TransCAD a través del proceso de lectura de estos archivos, descrito anteriormente.

En la ecuación 4 se muestra el modelo de gravedad utilizado para la etapa de distribución de viajes.

$$
T_{i j}^{p}=P_{i}^{p} \frac{A_{j}^{p} f\left(C_{i j}\right)}{\sum_{k=1}^{n} A_{k}^{p} f\left(C_{i j}\right)}
$$

donde:

$T_{i j}^{p} \quad$ : número de viajes de la zona i a la j para el propósito $\mathrm{p}$

$P_{i}^{p} \quad$ : número de viajes producidos en la zona i para el propósito $\mathrm{p}$ 
$\begin{array}{ll}A_{j}^{p} & \text { : número de viajes atraídos en la zona j para el } \\ & \text { propósito } \mathrm{p}\end{array}$

$C_{i j} \quad$ : costo generalizado de viajar entre las zonas ij

$f\left(C_{i j}\right)$ : función de impedancia para viajar entre las zonas ij

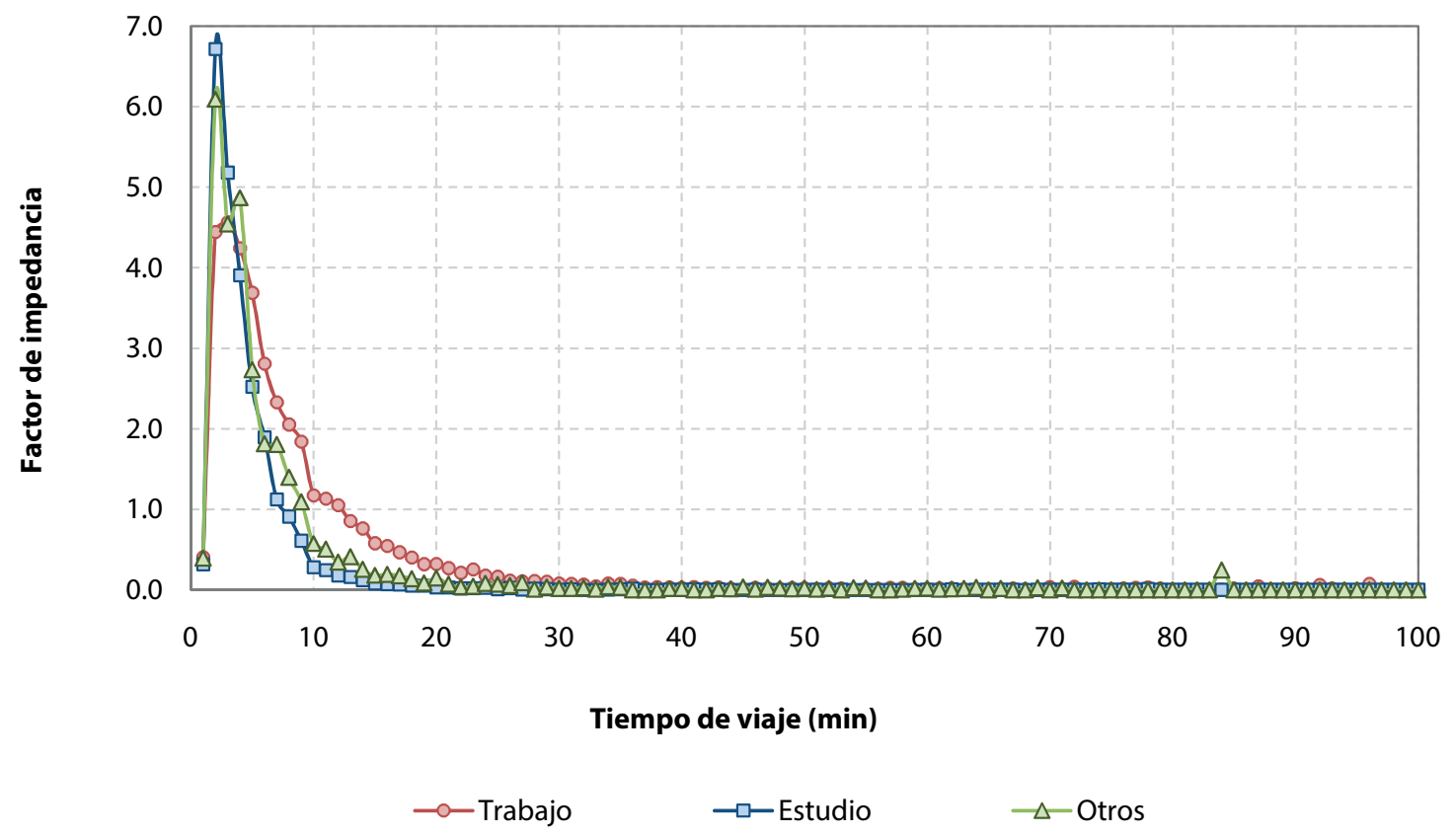

Figura 1. Funciones de impedancia por propósito de viaje

( L.C.R. Logística S.A., 2007)

\section{Selección Modal}

Se efectuó la selección modal a partir del modelo Logit Multinomial utilizado en el PRUGAM. El modelo logit utilizado requiere de dos insumos: la relación de costo percibido de viajar en transporte público con respecto al vehículo privado, y la posesión vehicular por zona, ambos en forma matricial.

En las ecuaciones 5, 6 y 7 se muestran las formulaciones Logit de selección modal por propósito de viaje.

$$
\begin{aligned}
P(T P)_{\text {trabajo }} & =\frac{e^{-11,32613 X_{1}-11,90138 X_{2}+13,12483}}{1+e^{-11,32613 X_{1}-11,90138 X_{2}+13,12483}} \\
P(T P)_{\text {estudio }} & =\frac{e^{-24,24499 X_{1}-5,63014 X_{2}+18,25250}}{1+e^{-24,24499 X_{1}-5,63014 X_{2}+18,25250}} \\
P(T P)_{\text {otros }} & =\frac{e^{-14,49212-4,85766 X_{2}+13,90893}}{1+e^{-14,49212 X_{1}-4,85766 X_{2}+13,90893}}
\end{aligned}
$$

donde:

$P$ : probabilidad de escogencia del transporte público

$X_{1}$ : relación entre los costos percibidos de viaje por transporte público con respecto al vehículo privado

$X_{2}$ : posesión vehicular de la zona de origen del viaje

$1-P(T P):$ probabilidad de escogencia del vehículo privado

\section{Asignación de Viajes en Vehículo Privado}

Para efectuar la asignación de viajes en vehículo privado fue necesario contar con la red de carreteras migrada y las propiedades por enlace: cantidad de carriles, velocidad y capacidad por sentido. Las características de la vía determinan los parámetros alfa y beta utilizados en la función cónica que permite simular el congestionamiento en las vías.

Para las asignaciones de flujos en Emme2, se utilizó una función cónica modificada, de manera que al superar relaciones volumen/ 
capacidad de 1,05, se desincentivara el uso de rutas congestionadas por medio de la incorporación de una asíntota en la función. TransCAD, sin embargo, tiene incorporadas diferentes bibliotecas de enlace dinámico (DLL) para la asignación de flujos, las cuales no son fácilmente modificables. Por lo tanto, se mantuvieron las funciones cónicas originales, sin la incorporación de la asíntota utilizada en Emme2.

El método de asignación utilizado en Emme2, Equilibrio del Usuario, es similar al que se encuentra disponible en TransCAD, Equilibrio del Usuario Conjugado, de tal forma que se lograron replicar los volúmenes asignados en el modelo original. La principal diferencia entre ambos métodos consiste en que, según Caliper (2015), el método Equilibrio del Usuario Conjugado requiere menor tiempo de procesamiento para alcanzar la convergencia. Por lo tanto, a pesar de que no fue posible modificar la función cónica, esto no representó un inconveniente.

\section{Asignación de Viajes en Transporte Público}

En la asignación de viajes en transporte público se utilizó la información asociada a las características operativas de las rutas de transporte público masivo (autobús y tren). Emme2 utiliza el método de Estrategias Óptimas, mientras que TransCAD utiliza el método de Equilibrium Pathfinder. La principal diferencia entre ambos métodos es que Estrategias Óptimas optimiza en términos del tiempo de viaje, por lo que no considera las tarifas para encontrar la ruta óptima del usuario, mientras que el método de Equilibrium Pathfinder optimiza en términos del costo generalizado y sí considera las tarifas. Sin embargo, esto no quiere decir que el modelo de PRUGAM establecido en Emme2 no considerara las tarifas dentro de su modelación, sino que se debió hacer un procesamiento adicional de los valores de tarifa para que se incluyeran como una demora adicional en los tiempos de viaje. Esta consideración adicional no es requerida en TransCAD y se pueden introducir las tarifas como una variable adicional del modelo.

Adicional al método utilizado para la asignación, se encontraron tres diferencias principales entre la modelación en Emme2 y TransCAD:

a) Los tiempos de viaje para cada ruta de transporte público son calculados automáticamente, con base en la longitud y velocidad de operación por enlace en Emme2. Sin embargo, TransCAD no calcula esos tiempos por defecto, por lo que es necesario calcularlos de previo a la asignación. Esto se efectúa mediante la planilla de cálculo de TransCAD, la cual permite determinar el tiempo de viaje para cada velocidad diferenciada por enlace. b) Las diferentes rutas de transporte público se agrupan con base en su velocidad de operación y cada grupo es considerado como un modo de transporte diferente. Se genera una tabla de modos, codificada con base en las indicaciones de TransCAD. Esta tabla es necesaria para considerar diferentes modos de viaje (tren y autobús) y velocidades distintas dentro de un mismo modo (rutas urbanas, rutas interurbanas, rutas expresas). Además, su función principal es asignar pesos y factores diferenciados por modo en la modelación, lo cual no era posible con Emme2.

c) Emme2 considera la capacidad del transporte público por modo (autobús o tren) y, según la frecuencia del servicio, estima la capacidad horaria. Por otra parte, TransCAD no calcula automáticamente el valor de capacidad horaria, motivo por el cual, previo a la asignación, mediante la planilla de cálculo fue necesario estimar la capacidad horaria para cada ruta de transporte público.

Debido a las diferencias mencionadas, la asignación de transporte público requiere de calibración adicional, la cual no fue necesaria en la asignación de vehículo privado. Por esta razón, los valores obtenidos de la asignación en Emme2 no pueden ser comparados de forma directa con los obtenidos en TransCAD.

\section{Modelo en lote (Batch)}

El proceso de modelación se puede efectuar tanto a través de la interfaz gráfica de TransCAD, como por medio de la generación de archivos en lote. Al igual que se había realizado con Emme 2 desde el 2007, en 2017 se programaron rutinas en lote (batch) en TransCAD, para así automatizar el análisis de distintos escenarios, de manera que se redujera la interacción del usuario con el sistema y se agilizara el proceso de generación de resultados. Es importante señalar que los códigos en Emme2 son diferentes a los de TransCAD, pues cada sistema maneja sus propios protocolos y formatos particulares, por lo que no son compatibles entre sí.

En Emme2, el código para recrear el modelo tuvo que ser generado en su totalidad a partir de la digitación de archivos de texto. Por otro lado, TransCAD permite la grabación de macros para cada etapa del modelo, realizadas a través de la interfaz gráfica, los cuales pueden ser modificados y procesados posteriormente.

A pesar de la facilidad de generar el código por medio de los macros en TransCAD, fue necesario programar algunos procesos intermedios de preparación de la base de datos. Estos procesos no podían ser generados a partir de la grabación de 
macros, ya que no corresponden a procedimientos asociados a las cuatro etapas de modelación de demanda de transporte urbano. Esto generó la necesidad de programar un paso intermedio entre la selección modal y la asignación de viajes, el cual permitiera agregar los viajes por propósito según el modo, así como agrupar los resultados de la asignación de vehículos privados de las zonas internas y externas, ya que éstos se generan de forma independiente. Por otro lado, y como se mencionó anteriormente, el proceso preestablecido por TransCAD para la etapa de generación de viajes no pudo ser adecuado para introducir las ecuaciones propias de producción de viajes, por lo que también tuvo que ser desarrollado de manera independiente de los macros.

A pesar de lo anterior, por medio del lenguaje en lote se pudo reproducir el modelo de cuatro etapas en su totalidad, con resultados satisfactorios para cada una de las etapas. El código generado en lote puede ser modificado para la evaluación de diferentes escenarios de asignación, tanto por cambios en la red de carreteras como en la red de transporte público. También puede ser modificado para reflejar variaciones en cualquiera de los parámetros asociados a las primeras tres etapas del modelo. Adicionalmente, permite las corridas de diferentes escenarios y años horizonte de manera consecutiva, sin necesidad de intervenciones adicionales.

\section{Resumen de Generalidades del Proceso de Migración}

En el cuadro 1 se resumen generalidades del proceso de migración del modelo, enfocado en la implementación de la metodología para aplicar los algoritmos de cada etapa y comparando los programas Emme2 y TransCAD 7.0.

Cuadro 1. Comparación por etapa del proceso de migración del modelo de demanda de transporte urbano de la GAM en Emme2 y TransCAD 7.0

\begin{tabular}{|c|c|c|}
\hline \multirow{2}{*}{ Etapa } & \multicolumn{2}{|c|}{ Software } \\
\hline & \multirow{3}{*}{$\begin{array}{l}\text { No cuenta con rutinas preelaboradas, sin embargo, } \\
\text { mediante arreglos matriciales y lenguaje de ma- } \\
\text { cros permite su implementación. }\end{array}$} & TransCAD 7.0 \\
\hline Generación de viajes & & \multirow{2}{*}{$\begin{array}{c}\text { Generado desde la interfaz gráfica en la planilla de cálculo } \\
\text { o mediante programación en lote (batch). }\end{array}$} \\
\hline Balanceo de viajes & & \\
\hline Distribución de viajes & $\begin{array}{c}\text { Se utiliza el método de Gravedad acotado en las } \\
\text { producciones, este fue implementado mediante } \\
\text { la programación en macros, ya que no cuenta con } \\
\text { rutinas preelaboradas. }\end{array}$ & $\begin{array}{l}\text { Se implementó desde el menú correspondiente al método } \\
\text { de Gravedad y se grabó el macro asociado al método para } \\
\text { correrlo como batch. }\end{array}$ \\
\hline Selección modal & $\begin{array}{l}\text { El método Logit Multinomial fue implementado } \\
\text { mediante programación en macros, ya que no } \\
\text { cuenta con rutinas preelaboradas. }\end{array}$ & $\begin{array}{c}\text { Se ejecutó el método Logit Multinomial desde el menú en } \\
\text { la interfaz gráfica y se grabó el macro asociado al método } \\
\text { para correrlo en lote. }\end{array}$ \\
\hline $\begin{array}{l}\text { Asignación de viajes en } \\
\text { vehículo privado }\end{array}$ & $\begin{array}{l}\text { El método utilizado fue Equilibrio del Usuario, el } \\
\text { cual se implementó desde la línea de comandos y } \\
\text { mediante programación en macros. }\end{array}$ & $\begin{array}{l}\text { El método utilizado fue Equilibrio del Usuario Conjugado, } \\
\text { el cual se ejecutó desde la interfaz gráfica mediante los } \\
\text { menús predeterminados y mediante programación en lote. }\end{array}$ \\
\hline $\begin{array}{l}\text { Asignación de viajes en } \\
\text { transporte público }\end{array}$ & $\begin{array}{l}\text { El método utilizado fue Estrategias Óptimas, el } \\
\text { cual se implementó desde la línea de comandos y } \\
\text { mediante programación en macros. }\end{array}$ & $\begin{array}{l}\text { El método utilizado fue Equilibrium Pathfinder, el cual } \\
\text { se ejecutó desde la interfaz gráfica mediante los menús } \\
\text { predeterminados y mediante programación en lote. }\end{array}$ \\
\hline
\end{tabular}

\section{CONCLUSIONES}

i. $\quad$ La base de datos completa de PRUGAM pudo migrarse de Emme2 a TransCAD, mediante diversos ajustes de forma, pero no de contenido en las variables, con excepción de la función cónica modificada, la cual tuvo que ser migrada en su forma original (sin asíntotas).

ii. Los métodos presentes en TransCAD son similares a los utilizados en Emme2 para el modelo de PRUGAM, por lo que el modelo de cuatro etapas del PRUGAM se pudo reproducir de manera satisfactoria en la plataforma TransCAD.

iii. Hasta que no se realice una nueva encuesta de hogares en la GAM, debidamente diseñada y aplicada, la base de datos del PRUGAM representa un insumo de gran valor para realizar las modelaciones de demanda de transporte urbano en la GAM.

iv. La migración de la base de datos de la red de carreteras, transporte público, información demográfica y 
socioeconómica, desde Emme2 hacia TransCAD, se realizó de forma considerablemente expedita por las facilidades de TransCAD para importar archivos en formato “.in”, correspondientes a Emme2.

v. TransCAD permite asignar pesos y factores diferenciados por modo para la asignación de viajes en transporte público, lo que permite modelar, de manera más realista, las diferencias percibidas por los usuarios entre los viajes en diferentes rutas de autobús y en tren. Por el contrario, Emme2 únicamente permitía definir parámetros globales para todas las rutas de transporte público.

vi. La interfaz gráfica de TransCAD 7.0 permite que usuarios inexpertos se familiaricen con programas de modelación de demanda. Por otra parte, mediante archivos en lote, los usuarios más experimentados pueden personalizar las diferentes fases del modelo de cuatro etapas, esto gracias a las capacidades de programación en lote existentes en TransCAD.
A la fecha de presentación del artículo, los autores se encontraban en el proceso de recopilación de datos de campo (aforos vehiculares y volúmenes movilizados en trasporte público) para iniciar con la calibración y actualización del modelo. Con base en esa información se procede a verificar que los valores correspondientes a la asignación en vehículo privado reflejan lo que ocurre en la realidad y se calibran los pesos asociados a los diferentes factores involucrados en la modelación de transporte público (tarifa, tiempo de espera, tiempo de viaje, etc.).

\section{AGRADECIMIENTOS}

Los autores desean manifestar su profundo agradecimiento a la Unidad de Seguridad Vial y Transportes del Programa de Infraestructura del Transporte (LanammeUCR) por su colaboración general, así como por facilitar el equipo necesario y las condiciones logísticas para el desarrollo del proyecto de investigación "Modelo de Demanda de Transporte Urbano en la GAM" (MDT-UCR).

\section{REFERENCIAS}

1. L.C.R. Logística S.A. (2007). Estudio de Oferta y Demanda de Transportes de la GAM. Tomo 1 Plan Regional Urbano de la Gran Área Metropolitana: Componente Movilidad y Transporte. Informe Final. Ministerio de Vivienda y Asentamientos Humanos. San José, Costa Rica.

2. Caliper, (2015). Manual de Usuario: Modelación de Demanda de Transporte con TransCAD vs 7.0. Estados Unidos. 